\title{
Superfluorescence from Lead Halide Perovskite Quantum Dot Superlattices
}

Gabriele Rainò ${ }^{1,2,3^{*}}$, Michael A. Becker ${ }^{3,4^{*}}$, Maryna I. Bodnarchuk ${ }^{2}$, Rainer F. Mahrt ${ }^{3}$, Maksym V. Kovalenko ${ }^{1,2}$, and Thilo Stöferle ${ }^{3}$

${ }^{1}$ Institute of Inorganic Chemistry, Department of Chemistry and Applied Bioscience, ETH Zurich, 8093 Zurich, Switzerland.

${ }^{2}$ Laboratory of Thin Films and Photovoltaics, Empa - Swiss Federal Laboratories for Materials Science and Technology, 8600 Dübendorf, Switzerland.

${ }^{3}$ IBM Research - Zurich, Säumerstrasse 4, 8803 Rüschlikon, Switzerland.

${ }^{4}$ Optical Materials Engineering Laboratory, ETH Zurich, 8092 Zurich, Switzerland.

*These authors contributed equally to this work.

An ensemble of emitters can behave significantly different from its individual constituents when interacting coherently via a common light field. After excitation, collective coupling gives rise to an intriguing many-body quantum phenomenon, resulting in short, intense bursts of light: so-called superfluorescence ${ }^{1}$. Because it requires a fine balance of interactions between the emitters and their decoupling from the environment, together with close identity of the individual emitters, superfluorescence has thus far been observed only in a limited number of very specific systems, such as certain atomic and molecular gases and a few select solid-state systems $^{2-7}$. With colloidal nanocrystals, however, which are increasingly recognized as bright, practically suited photonic sources for optoelectronics ${ }^{8,9}$, the generation of superfluorescent light was precluded by inhomogeneous emission broadening, low oscillator strength, and fast exciton dephasing. Using caesium lead halide (CsPbX, $\mathrm{X}=$ $\mathrm{CI}, \mathrm{Br}$ ) perovskite nanocrystals ${ }^{10-13}$ that are self-organized into highly ordered threedimensional superlattices allows us to observe key signatures of superfluorescence: dynamically red-shifted emission with more than twenty-fold accelerated radiative decay, extension of the first-order coherence time by more than a factor of four, 
photon bunching, and delayed emission pulses with Burnham-Chiao ringing behaviour $^{14}$ at high excitation density. These mesoscopically extended coherent states can be employed to boost opto-electronic device performances ${ }^{15}$ and enable entangled multi-photon quantum light sources ${ }^{16,17}$.

Spontaneous emission (SE) of photons, such as fluorescence commonly used in displays or lighting, occurs due to coupling excited two-level systems (TLS) to the vacuum modes of the electromagnetic field, effectively stimulated by its zero-point fluctuations. In 1954, R. H. Dicke predicted ${ }^{18}$ that an ensemble of $N$ identical TLS confined in a volume smaller than $\sim \lambda^{3}$ ( $\lambda$ is the corresponding emission wavelength of the TLS) can exhibit coherent and cooperative spontaneous emission. This so-called superradiant emission results from the coherent coupling between individual TLS through the common vacuum modes, effectively leading to a single giant emitting dipole from all participating TLS. Superradiant emission has been observed in distinctly different physical systems, such as molecular aggregates and crystals $^{19}$, nitrogen vacancy centres in diamond ${ }^{20}$ and epitaxially grown quantum dots ${ }^{21}$. In the case when the excited TLS are initially fully uncorrelated, the coherence can be established only through spontaneously triggered correlations due to quantum fluctuations rather than by coherent excitation. When this occurs, a so-called superfluorescent (SF) pulse is emitted ${ }^{1}$ (Figure 1, illustrated for the present study). Both superradiant emission and coherent SF bursts are characterized by an accelerated radiative decay time $\tau_{\mathrm{SF}} \sim \tau_{\mathrm{SE}} / N$, where the exponential decay time $\tau_{\mathrm{SE}}$ of the uncoupled TLS is shortened by the number of coupled emitters $N$. In addition, SF exhibits the following fundamental signatures, which magnitudes are also dependent on the excitation density: (i) a delay or build-up time $\tau_{\mathrm{D}} \sim \ln (N) / N$ during which the emitters couple and phase-synchronize to each other, and which corresponds to the time delay between the excitation and onset of the cooperative emission (Figure 1) and (ii) coherent Rabi-type oscillations in the time domain due to the strong light-matter interaction, known as Burnham-Chiao ringing ${ }^{14,22}$.

SF was first observed in a dense gas of hydrogen fluoride ${ }^{2}$, followed by a limited number of solid-state systems, such as $\mathrm{CuCl}$ nanocrystals (NCs) formed in a $\mathrm{NaCl}$ matrix ${ }^{4}$, $\mathrm{KCl}$ crystals doped with peroxide anions $\left(\mathrm{O}_{2}^{-}\right)\left(\right.$ref. $\left.^{3}\right)$, and some select semiconductor crystals (ZnTe and InGaAs/GaAs multi-quantum wells) $)^{5,6}$. Practical implementation of such an enhanced radiative property is a persistent challenge. Besides stringent requirements for the 
emissive material (e.g., high oscillator strength, small inhomogeneous line-broadening, small exciton dephasing), equally important are structural, optical and device engineerability. Colloidal semiconductor NCs, also known as colloidal quantum dots (QDs), could fill this gap as they are structurally and optically versatile, and highly suited for the entire visible spectral range. While they are actively pursued for photonic applications ${ }^{8,9,23}$, they have not been reported to exhibit SF.

Here, we use colloidal NCs of caesium lead halide perovskites ( $\mathrm{CsPbX}_{3}, \mathrm{X}=\mathrm{Cl}, \mathrm{Br}$ ) that can be synthesized with narrow size dispersion and are known to exhibit moderate quantum confinement effects, resulting in narrow-band emission combined with exceptionally large oscillator strength from a bright triplet state ${ }^{10,11,24}$. In order to foster cooperative behaviour, we employ structurally well-defined, long-range ordered, and densely packed arrays of such NCs, known as superlattices, produced by means of solvent-drying-induced spontaneous assembly ${ }^{25-28}$. Similarly, regular arrays of II-VI semiconductor NCs have been used to obtain collective effects in the electronic domain, i.e., band-like transport ${ }^{15}$. Figure $2 a$ outlines the superlattice formation (see also Methods), using a solution of highly monodispersed $\mathrm{CsPbBr}_{3} \mathrm{NCs}$ with a mean size of $9.5 \mathrm{~nm}$ and standard size-deviation of less than 5\% (Extended Data Figure 1). In the self-assembly process, cubic individual superlattice domains are formed (i.e., supercrystals), each consisting of up to several millions of NCs. Optical microscopy (Figure 2c) reveals superlattices with a lateral size of up to $5 \mu \mathrm{m}$, randomly distributed in a uniform film on a $5 \times 7 \mathrm{~mm}$ sample (Figure 2d). Transmission electron microscopy confirms that highly ordered superlattices consist of well-separated individual NCs (Figure 2e and Extended Data Figure 2). More details of the self-assembly process are reported in the Methods section.

Figure 3a displays the photoluminescence (PL) spectrum of a single $\mathrm{CsPbBr}_{3}$ superlattice (excited at $3.06 \mathrm{eV}$ ) exhibiting two emission peaks. This and all other optical measurements were performed at a temperature of $6 \mathrm{~K}$ in vacuum or Helium atmosphere (see Methods for details). The high-energy emission peak coincides with the centre energy of the disordered dense film of $\mathrm{CsPbBr}_{3} \mathrm{NCs}$ (glassy state) and is therefore assigned to noncoupled QDs. In addition, a narrow, red-shifted emission peak appears in superlattices, which we assign to the emission of coupled QDs, which is best fitted with a Lorentzian (full width at half maximum, FWHM coupled $=11 \mathrm{meV}$ ). The peaks of the uncoupled QDs in a superlattice and 
in the glassy films are best fitted with a Gaussian, as expected for disordered ensembles. The width of the uncoupled QDs (FWHM uncoupled $=55 \mathrm{meV}$ ) is slightly broader than the one of the amorphous film ( $F W H M_{\text {amorphous }}=35 \mathrm{meV}$ ), which can be explained assuming that the more "identical QDs" within the superlattice are now forming the peak of coupled QDs while the remaining uncoupled ones appear more disordered than the inhomogeneous energy distribution of the primary QD material. We can exclude that the red-shifted feature, which is at $\sim 70 \mathrm{meV}$ lower energy than the uncoupled QD emission, originates from the emission from trions, bi-excitons or multi-excitons, because their energy shifts reportedly are 10-20 meV (refs. ${ }^{11,12}$ ), and these would be observable in the disordered ensemble, too. The number and interaction strength of coupled QDs determine the magnitude of the energetic shift. Statistics from 10 superlattices from different samples give an average static red-shift of $(64 \pm 6) \mathrm{meV}$, average $\mathrm{FWHM}_{\text {coupled }}=(15 \pm 4) \mathrm{meV}$ and average $\mathrm{FWHM}_{\text {uncoupled }}=(49 \pm 21) \mathrm{meV}$. In most superlattices, we observe a sub-structure in this red-shifted emission band, which we attribute to the presence of several, slightly different independent domains within the same individual superlattice.

A central feature of the cooperative emission is the modification of the radiative lifetime $^{18}$, as demonstrated experimentally with several quantum emitters ${ }^{6,20,21}$. In timeresolved PL decay measurements, at a very low excitation fluence $\left(5 \mathrm{~nJ} / \mathrm{cm}^{2}\right)$, we do not observe a significant modification of the decay of the coupled QD emission compared to the uncoupled QD emission (Figure $3 b$ inset). The absence of accelerated emission at vanishing excitation density and the presence of the red-shifted feature also in PL excitation scans (Extended Data Figure 3) corroborates that the static $\sim 70 \mathrm{meV}$ red-shift originates from incoherent coupling of the QDs in the ground state, similar to various molecular aggregates ${ }^{19}$. Already at a slightly higher excitation density, we observe an accelerated $\mathrm{PL}$ decay of the coupled QD emission peak in comparison to the PL decay of uncoupled QDs with 1/e decay times of $\tau_{\mathrm{SF}}=148 \mathrm{ps}$ and $\tau_{\mathrm{QD}}=400 \mathrm{ps}$, respectively, for an excitation density of $500 \mathrm{~nJ} / \mathrm{cm}^{2}$ per pulse (Figure $3 b$ ). In contrast to the predominantly mono-exponential decay of the uncoupled QDs, this SF emission decay is approximated well by a stretched exponential ${ }^{29}$ (see Methods section), because the number of excited coupled emitters, and therefore the emission acceleration, varies during the decay. Furthermore, in contrast to the uncoupled QDs, the SF decay time is strongly dependent on excitation power (inset Figure $3 \mathrm{~b}$ ) because 
it scales with the coupling strength among the QDs, given by the intensity in the common light-field that effectively corresponds to a change in the number of coherently coupled QDs. When the spectrally and temporally integrated emission is fitted with a power law, we obtain an exponent of 1 (Extended Data Figure 4), indicating that excitation density-dependent nonradiative decay channels (e.g. Auger recombination) are absent. Notably, no threshold behaviour as for amplified spontaneous emission (ASE) is observed.

The cooperative emission process strongly influences the coherence of the emitted light. First-order correlation measurements of each of the two emission peaks by means of a Michelson interferometer allow us to monitor the interference pattern and therefore the phase coherence time (Figure 4a). The emission band of the uncoupled QDs exhibits $38 \mathrm{fs}$ coherence time, best fitted with a Gaussian decay (Figure 4a, upper graph), typical of incoherent (thermal) light sources. The emission from the coherently coupled QDs (Figure 4a, lower graph) exhibits a much longer coherence time with an exponential decay of $140 \mathrm{fs}$. For some superlattices, a Gaussian decay is observed (Extended Data Figure 5a), which might be attributed to number fluctuations within the coherent SF state ${ }^{30}$.

Second-order coherence of the emitted light is evinced by the statistics of the photon arrival time on a detector ${ }^{31}$. Typical coherent light, as from a laser, shows a random distribution (Poissonian) of photon arrival times, while a single TLS exhibits photon antibunching (sub-Poissonian distribution). In contrast, the cooperative emission from coupled QDs leads to coherent multi-photon emission bursts. Figure 4 reports the second-order correlation function, $g^{(2)}(\tau)=\frac{\langle I(t) I(t+\tau)\rangle}{\langle I(t)\rangle^{2}}$ for both PL emission bands, where $I(t)$ is the signal intensity at time $t$. For the uncoupled QD emission (Figure $4 \mathrm{~b}$, upper graph), a flat $g^{(2)}(\tau)=1$ is observed because the experimental temporal resolution ( $40 \mathrm{ps})$ is insufficient to resolve the expected thermal bunching. The SF emission band, however, shows pronounced photon bunching (Figure 4b, lower graph) because the coherent coupling leads to the correlated emission of multiple photons within a short time interval. Photon bunching is only observable in superlattices with single or a few SF domains, i.e., where no sub-structure is visible in the red-shifted emission band, because spectrally overlapping uncorrelated aggregated domains within the same superlattice reduce the bunching peak's visibility, as predicted by theory ${ }^{32}$. Yet, it is a robust effect that is observed with pulsed excitation and for mixed-halide 
( $\mathrm{CsPbBr}_{2} \mathrm{Cl}$, emitting at higher energies) QD superlattices, too (see Extended Data Figure $5 \mathrm{~b}$ and $6 \mathrm{~b}$, respectively). Remarkably, some superlattices with supposedly well-isolated coherently coupled QDs exhibit $g^{(2)}(\tau)>2$ (inset Figure 4), similar to superthermal emission ${ }^{31}$. The exponential decay time of the second-order correlation is of the order of the radiative decay of the SF emission for low excitation densities $\left(\tau_{g^{(2)}}=224 \mathrm{ps}\right)$.

Very distinct characteristics of SF emission concern the time evolution of the emitted light under strong driving conditions. Figure 5a shows a streak camera image acquired at an excitation density of $600 \mu \mathrm{J} / \mathrm{cm}^{2}$, where in addition to a drastically shortened radiative decay, a finite rise time and subsequent oscillations of the emission are observed. Quantitative analysis on spectrally integrated PL decay traces for various excitation power densities is shown in Figure $5 b$ (for details see Methods). As a function of the excitation density, the decay time shortens to $14 \mathrm{ps}$ (Figure $5 \mathrm{c}$, upper panel). From this decay shortening, which is an order of magnitude stronger than reported for the collective emission from other QD systems ${ }^{4,21}$, we can estimate an average number of coherently coupled QDs to be $N \sim 28$. This is only an effective value and a conservative estimate, because the energetic disorder of the QD emission energies ( $F W H M_{\text {coupled }}=11 \mathrm{meV}$ ) still exceeds significantly the emission peak width of individual QDs (typically FWHM $\sim 1 \mathrm{meV}$, ref. ${ }^{11}$ ) and thereby effectively reduces the SF coupling ${ }^{33}$. The SF emission experiences a dynamical red-shift of up to 15 $\mathrm{meV}$ due to renormalization of the emission energy from the coherent coupling ${ }^{7}$, which decreases in the course of the decay as the number of excitations reduces (Extended Data Figure 7). The peak intensity increases super-linearly over three orders of magnitude (Figure $5 \mathrm{c}$, middle panel), according to a power-law dependence with an exponent of $\alpha=1.5 \pm 0.1$, deviating from the theoretically expected value of $\alpha=2$ (ref. ${ }^{7}$ ), presumably due to saturation effects $^{5}$. Nevertheless, no significant quenching effects of the emission for high excitation powers were found, verifying that the decay remains essentially radiative (Extended Data Figure $4 d)$. Furthermore, a shortening of the SF delay time $\left(\tau_{\mathrm{D}}\right)$, after which the photon burst is emitted, is observed (Figure 5c, bottom panel). This characteristic of SF is a consequence of the time it takes for phase-locking the individual dipoles and scales with the number $N$ of excited coupled QDs according to $\tau_{\mathrm{D}} \sim \frac{\log (N)}{N}$ (see Methods section). 
As SF crucially depends on low decoherence and low inhomogeneous spread, it should be noted that SF coupling is strongly affected by the environment around the QDs (number of free ligands), the superlattice assembly, and by the quality of the QDs themselves. Thus, while a large fraction of the superlattices displays a red-shifted peak from the coupled QD emission, the amount of photon-bunching and Burnham-Chiao ringing varied from superlattice to superlattice. However, experiments employing different batches of NCs and superlattice assemblies of $\mathrm{CsPbBr}_{3}$ and $\mathrm{CsPbBr}_{2} \mathrm{Cl} \mathrm{NCs}$ (see Extended Data Figures 6-9) were consistently reproducible, but further optimization of the synthesis and assembly is likely to improve the yield of SF domains. It is important to note that experiments on control samples with diluted, uncoupled QDs under similar excitation conditions do not show any of these signatures of SF (Extended Data Figure 10), proving that it is a genuine multi-particle effect.

Our measurements reveal that coherent SF coupling can be achieved in long-range ordered self-assembled superlattices of fully inorganic $\mathrm{CsPbX}_{3}$ perovskite NCs, resulting in strong emission bursts. Colloidal NCs and their assemblies have proven to be excellent building blocks for a large variety of opto-electronic devices, and these cooperative effects now allow modification of the opto-electronic properties beyond what is possible on the individual QD level with chemical engineering approaches. This opens up new opportunities for high-brightness and multi-photon quantum light sources, and could enable the exploitation of cooperative effects for long-range quantum transport and ultra-narrow tuneable lasers.

\section{References}

1 Bonifacio, R. \& Lugiato, L. A. Cooperative radiation processes in two-level systems: Superfluorescence. Physical Review A 11, 1507-1521 (1975).

2 Skribanowitz, N., Herman, I. P., MacGillivray, J. C. \& Feld, M. S. Observation of Dicke Superradiance in Optically Pumped HF Gas. Physical Review Letters 30, 309-312 (1973).

3 Malcuit, M. S., Maki, J. J., Simkin, D. J. \& Boyd, R. W. Transition from superfluorescence to amplified spontaneous emission. Physical Review Letters 59, 1189-1192 (1987).

4 Miyajima, K., Kagotani, Y., Saito, S., Ashida, M. \& Itoh, T. Superfluorescent pulsed emission from biexcitons in an ensemble of semiconductor quantum dots. Journal of Physics: Condensed Matter 21, 195802 (2009).

5 Dai, D. C. \& Monkman, A. P. Observation of superfluorescence from a quantum ensemble of coherent excitons in a ZnTe crystal: Evidence for spontaneous Bose-Einstein condensation of excitons. Physical Review B 84, 115206 (2011). 
Noe II, G. T. et al. Giant superfluorescent bursts from a semiconductor magneto-plasma. Nature Physics 8, 219-224 (2012).

7 Cong, K. et al. Dicke superradiance in solids. J. Opt. Soc. Am. B 33, C80-C101 (2016).

8 Talapin, D. V., Lee, J.-S., Kovalenko, M. V. \& Shevchenko, E. V. Prospects of Colloidal Nanocrystals for Electronic and Optoelectronic Applications. Chemical Reviews 110, 389-458 (2010).

$9 \quad$ Kovalenko, M. V. et al. Prospects of Nanoscience with Nanocrystals. ACS Nano 9, 1012-1057 (2015).

10 Protesescu, L. et al. Nanocrystals of Cesium Lead Halide Perovskites (CsPbX3, X $=\mathrm{Cl}, \mathrm{Br}$, and I): Novel Optoelectronic Materials Showing Bright Emission with Wide Color Gamut. Nano Letters 15, 3692-3696 (2015).

11 Rainò, G. et al. Single Cesium Lead Halide Perovskite Nanocrystals at Low Temperature: Fast SinglePhoton Emission, Reduced Blinking, and Exciton Fine Structure. ACS Nano 10, 2485-2490 (2016).

$12 \mathrm{Fu}, \mathrm{M}$. et al. Neutral and Charged Exciton Fine Structure in Single Lead Halide Perovskite Nanocrystals Revealed by Magneto-optical Spectroscopy. Nano Letters 17, 2895-2901 (2017).

13 Kovalenko, M. V., Protesescu, L. \& Bodnarchuk, M. I. Properties and potential optoelectronic applications of lead halide perovskite nanocrystals. Science 358, 745-750 (2017).

14 Burnham, D. C. \& Chiao, R. Y. Coherent Resonance Fluorescence Excited by Short Light Pulses. Physical Review 188, 667-675 (1969).

15 Kagan, C. R. \& Murray, C. B. Charge transport in strongly coupled quantum dot solids. Nature Nanotechnology 10, 1013-1026 (2015).

16 Afek, I., Ambar, O. \& Silberberg, Y. High-NOON States by Mixing Quantum and Classical Light. Science 328, 879 (2010).

17 Munoz, C. S. et al. Emitters of N-photon bundles. Nature Photonics 8, 550-555 (2014).

18 Dicke, R. H. Coherence in Spontaneous Radiation Processes. Physical Review 93, 99-110 (1954).

19 Spano, F. C. The Spectral Signatures of Frenkel Polarons in H- and J-Aggregates. Accounts of Chemical Research 43, 429-439 (2010).

20 Bradac, C. et al. Room-temperature spontaneous superradiance from single diamond nanocrystals. Nature Communications 8, 1205 (2017).

21 Scheibner, M. et al. Superradiance of quantum dots. Nature Physics 3, 106-110 (2007).

22 Heinzen, D. J., Thomas, J. E. \& Feld, M. S. Coherent Ringing in Superfluorescence. Physical Review Letters 54, 677-680 (1985).

23 Shirasaki, Y., Supran, G. J., Bawendi, M. G. \& Bulović, V. Emergence of colloidal quantum-dot lightemitting technologies. Nature Photonics 7, 13 (2012).

24 Becker, M. A. et al. Bright triplet excitons in caesium lead halide perovskites. Nature 553, 189 (2018).

25 Boles, M. A., Engel, M. \& Talapin, D. V. Self-Assembly of Colloidal Nanocrystals: From Intricate Structures to Functional Materials. Chemical Reviews 116, 11220-11289 (2016).

26 Murray, C. B., Kagan, C. R. \& Bawendi, M. G. Synthesis and Characterization of Monodisperse Nanocrystals and Close-Packed Nanocrystal Assemblies. Annual Review of Materials Science 30, 545610 (2000).

27 Geuchies, J. J. et al. In situ study of the formation mechanism of two-dimensional superlattices from PbSe nanocrystals. Nature Materials 15, 1248 (2016).

28 Nagaoka, Y. et al. Nanocube Superlattices of Cesium Lead Bromide Perovskites and Pressure-Induced Phase Transformations at Atomic and Mesoscale Levels. Advanced Materials 29, 1606666 (2017).

29 Temnov, V. V. \& Woggon, U. Superradiance and Subradiance in an Inhomogeneously Broadened Ensemble of Two-Level Systems Coupled to a Low-Q Cavity. Physical Review Letters 95, 243602 (2005).

30 Whittaker, D. M. \& Eastham, P. R. Coherence properties of the microcavity polariton condensate. Europhysics Letters 87, 27002 (2009).

31 Jahnke, F. et al. Giant photon bunching, superradiant pulse emission and excitation trapping in quantum-dot nanolasers. Nature Communications 7, 11540 (2016). 
Temnov, V. V. \& Woggon, U. Photon statistics in the cooperative spontaneous emission. Optics Express 17, 5774 (2009). Ishikawa, A., Miyajima, K., Ashida, M., Itoh, T. \& Ishihara, H. Theory of Superfluorescence in Highly Inhomogeneous Quantum Systems. Journal of the Physical Society of Japan 85, 034703 (2016).

\section{Main Figure Legends}

Figure 1 | Schematic of the build-up process of SF. An initially uncorrelated ensemble of TLS (randomly oriented green arrows) is excited by a light pulse (blue arrow). After time $\tau_{\mathrm{D}}$ their phases are synchronized (aligned green arrows) such that they cooperatively emit a SF light pulse (red arrow). Grey cubes represent longrange ordered self-assembled superlattices.

Figure 2 | Formation of $\mathrm{CsPbX}_{3}(\mathrm{X}=\mathrm{Cl}, \mathrm{Br}) \mathrm{NC}$ superlattices by drying-mediated self-assembly. a, Illustration of the assembly process. b. High-resolution scanning transmission electron microscopy image (HAADF-STEM) of a single CsPbBr 3 NC. c, Optical microscope image and d, photograph (under UV light) of a layer of micron-sized, three-dimensional, cubic-shaped NC superlattices. e, HAADF-STEM image of a single superlattice of $\mathrm{CsPbBr}_{3} \mathrm{NCs}$. The cubic shape of the individual perovskite NC building blocks is translated into the symmetry of the superlattice (simple cubic packing). The inset shows a zoom-in where the individual NCs are visible.

Figure 3 | Optical properties of $\mathrm{CsPbBr}_{3}$ QD superlattices. a, $\mathrm{PL}$ spectrum of a single $\mathrm{CsPbBr}_{3}$ superlattice (black solid line). The high-energy band is assigned to the emission of uncoupled QDs. The low-energy band is the result of the emission of coupled QDs and is not present in glassy films of NCs (green solid line). The shaded areas are fits to the data (see main text). b. Time-resolved PL decay of the two emission bands at $500 \mathrm{~nJ} / \mathrm{cm}^{2}$ excitation density after applying suitable spectral filters to separate the two components. With increasing excitation density, the decay from the coherently coupled QDs is significantly faster than from the uncoupled ones. The inset shows the power-dependence of the 1/e-decay times of both components.

Figure 4 | First- and second-order coherence properties of $\mathrm{CsPbBr}_{3}$ QD superlattices. a, First-order correlation of the two emission bands as obtained from the interference fringe visibility using a Michelson interferometer. The high-energy band of the uncoupled QDs has a very short phase coherence time $(<40 \mathrm{fs}$, upper graph), whereas the red-shifted band from the coupled QDs is characterized by much longer phase coherence (140 fs, lower graph). The solid lines are fits to the data (see text). The inset shows an example of the real space interferogram. b, Second-order correlation function, $g^{(2)}(\tau)$, obtained with a Hanbury-Brown and Twiss setup in start-stop configuration. For the high-energy band (upper graph), a flat profile with $g^{(2)}(\tau)=1$ is observed. The red-shifted emission band (lower graph) from the SF emission displays a pronounced bunching peak, characteristic of the correlated emission during a photon burst. The data are fitted to the function $g^{(2)}(\tau)=$ $1+A \cdot \exp \left(-\left(\left|\tau-\tau_{0}\right| / \tau_{c}\right)\right.$ (solid lines). The inset shows an example of superbunching with $g^{(2)}(0)>2$ from a single superlattice.

Figure 5 | Burnham-Chiao ringing behaviour of $\mathrm{CsPbBr}_{3}$ QD superlattices. a, Streak camera image of SF dynamics obtained with high excitation density of $600 \mu \mathrm{J} / \mathrm{cm}^{2}$. b. Extracted time-resolved emission intensity traces for five different excitation powers. Solid red lines are weighted best-fits to a model that employs a biexponential decay function with damped oscillations. c, Top: Effective SF decay (blue circles) as a function of the excitation power density fitted according to the SF model (solid blue line). Middle: Red circles represent the peak SF emission intensity that increases super-linearly with excitation power, corresponding to a power-law dependence with an exponent $\alpha=1.5 \pm 0.1$ (solid dark-red line). Bottom: The extracted delay time $\tau_{\mathrm{D}}$ (green 
313 circles) decreases at high excitation power due to the increased interaction among the emitters. The green solid

314 line is the best fit according the model described in the Methods section. The error bars represent the 315 parameters' fit uncertainty.

316

317 
319 Synthesis of $\mathrm{CsPbBr}_{3}$ nanocrystals. In a $25 \mathrm{ml}$ three-necked flask, $\mathrm{PbBr}_{2}$ (69 mg, 0.188 $320 \mathrm{mmol}$, Aldrich, 99\%) was suspended in octadecene $(5 \mathrm{ml})$, dried at $100^{\circ} \mathrm{C}$ for $30 \mathrm{~min}$, and mixed with oleic acid $\left(0.5 \mathrm{ml}\right.$, vacuum-dried at $\left.100^{\circ} \mathrm{C}\right)$ and oleylamine $(0.5 \mathrm{ml}$ vacuum-dried at $100^{\circ} \mathrm{C}$ ). When $\mathrm{PbBr}_{2}$ was dissolved, the reaction mixture was heated up to $180^{\circ} \mathrm{C}$ and preheated caesium oleate in octadecene $(0.4 \mathrm{ml}, 0.125 \mathrm{M})$ was injected. The reaction mixture was cooled immediately with an ice bath to room temperature.

Synthesis of $\mathrm{CsPbBr}_{2} \mathrm{Cl}$ nanocrystals. In a $25 \mathrm{ml}$ three-necked flask, $\mathrm{PbBr}_{2}$ (45 mg, 0.12 mmol, Aldrich, 99\%), $\mathrm{PbCl}_{2}$ (18 mg, $0.064 \mathrm{mmol}, \mathrm{ABCR}$ ) and $1 \mathrm{ml}$ trioctylphosphine (Strem, $97 \%$ ) was suspended in octadecene $(5 \mathrm{ml})$, dried at $100^{\circ} \mathrm{C}$ for $30 \mathrm{~min}$, and mixed with oleic acid $\left(0.5 \mathrm{ml}\right.$, vacuum-dried at $\left.100^{\circ} \mathrm{C}\right)$ and oleylamine $\left(0.5 \mathrm{ml}\right.$ vacuum-dried at $\left.100^{\circ} \mathrm{C}\right)$. When $\mathrm{PbCl}_{2}$ and $\mathrm{PbBr}_{2}$ were dissolved, the reaction mixture was heated up to $180^{\circ} \mathrm{C}$ and preheated caesium oleate in octadecene $(0.4 \mathrm{ml}, 0.125 \mathrm{M})$ was injected. The reaction mixture was cooled immediately with an ice bath to room temperature.

Purification and size-selection of $\mathrm{CsPbX}_{3}(X=\mathrm{Cl}, \mathrm{Br})$ nanocrystals. A critical factor for self-assembly of cubic-shaped $\mathrm{CsPbX}_{3} \mathrm{NCs}$ is to start with an initially high level of monodispersity. The crude solution was centrifuged at $12100 \mathrm{rpm}$ for $5 \mathrm{~min}$, following which the supernatant was discarded, and the precipitate was dissolved in $300 \mu$ hexane. The hexane solution was centrifuged again and the precipitate was discarded. The supernatant was diluted two times and used for further purification. Subsequently, two methods of purification of the NCs were applied: (a) $50 \mu$ l hexane, $0.6 \mu$ l oleic acid, and $0.6 \mu l$ oleylamine were added to $50 \mu \mathrm{l} \mathrm{NCs} \mathrm{in} \mathrm{hexane.} \mathrm{The} \mathrm{colloid} \mathrm{was} \mathrm{destabilized} \mathrm{by} \mathrm{adding} 50 \mu l$ acetone, followed by centrifuging and dispersing the NCs in $300 \mu \mathrm{l}$ toluene. This solution was used further for the preparation of the 3D-superlattices. (b) $50 \mu \mathrm{l}$ hexane and $100 \mu$ l toluene were added to $50 \mu \mathrm{l} \mathrm{NCs} \mathrm{in} \mathrm{hexane.} \mathrm{The} \mathrm{colloid} \mathrm{was} \mathrm{destabilized} \mathrm{by} \mathrm{adding} 50 \mu$ acetonitrile, followed by centrifuging and dispersing the NCs in $300 \mu l$ toluene. This solution was used further for the preparation of the 3D-superlattices.

Preparation of 3D-superlattices. $\mathrm{CsPbX}_{3} \mathrm{NC}$ superlattices were prepared on glass or on $5 \times$ $7 \mathrm{~mm}$ silicon substrates. Shortly before the self-assembly process, the silicon substrate was dipped into $4 \%$ solution of HF in water for $1 \mathrm{~min}$, followed by washing with water. In a typical assembly process, the substrate was placed in a $10 \times 10 \times 10 \mathrm{~mm}$ Teflon well and $10 \mu \mathrm{l}$ of 
slide and the toluene was then allowed to evaporate slowly. 3D-superlattices of $\mathrm{CsPbBr}_{3} \mathrm{NCs}$ were formed upon complete evaporation of the toluene. Typical lateral dimensions of individual superlattices ranged from 1 to $10 \mu \mathrm{m}$ wherein some of them arrange into clusters of several superlattices and others remain spatially well-isolated so that PL measurements can be performed on an individual superlattice.More intense purification or greater polydispersity of NCs led to disordered or 2D assemblies (glassy films). Furthermore, the formation of NC superlattices can serve to further narrow the size distribution and shape uniformity within the ensemble (with smaller or larger NCs being repelled from the NC domain), especially in the case of simple cubic packing of cubes, which is particularly intolerant to size and shape variations.

Optical spectroscopy. All measurements were performed at cryogenic temperatures $(6 \mathrm{~K})$. For $\mathrm{PL}$, time-resolved $\mathrm{PL}$, and second-order photon-correlation measurements on single superlattices, the sample was mounted in an evacuated liquid-helium flow cryostat on $x y z$ positioning stages and excited with a fibre-coupled excitation laser at an energy of $3.06 \mathrm{eV}$, either in continuous wave mode or pulsed mode with $40 \mathrm{MHz}$ repetition rate (pulse duration $50 \mathrm{ps}$ ). The excitation laser was filtered with a short-pass filter and directed towards the longworking distance $100 \times$ microscope objective (numerical aperture $N A=0.7$ ) by a dichroic beam splitter, resulting in a nearly Gaussian-shaped excitation spot with $1 / e^{2}$ radius of 1.4 $\mu \mathrm{m}$. The emission was collected via the same microscope objective and filtered using a tuneable bandpass filter. For PL measurements, the collected light was then dispersed by a 300 lines $/ \mathrm{mm}$ grating inside a $750 \mathrm{~mm}$ monochromator and detected by an EMCCD camera. For measurements of the PL decay, we filtered the emission with a tuneable band-pass $(\mathrm{FWHM}=15 \mathrm{~nm})$ and recorded the decay with an avalanche photo diode single photon detector with a time resolution of 30 ps connected to a time-correlated single-photon-counting system. The photon correlation was recorded using a similar setup with two detectors in a Hanbury-Brown-Twiss setup configuration.

To record streak camera images and first-order coherence measurements, we excited the sample, which was mounted in an exchange-gas cryostat at $6 \mathrm{~K}$, with a frequency-doubled regenerative amplifier seeded with a mode-locked Ti:sapphire laser with a pulse duration of 100-200 fs and a repetition rate of $1 \mathrm{kHz}$ at $3.1 \mathrm{eV}$. For both excitation and detection, we used an $80 \mathrm{~mm}$ lens $(N A=0.013$ after iris), resulting in an excitation spot area of $20 \times 40 \mu \mathrm{m}$. 
381

The recorded PL was dispersed by a grating with 150 lines $/ \mathrm{mm}$ in a $300 \mathrm{~mm}$ spectrograph and detected with a streak camera with a nominal time resolution of 2 ps and an instrument response function FWHM of 4 ps (see Extended Data Figure 10). First-order coherence measurements were performed using a Michelson interferometer. Here a non-polarizing beam splitter is used to split and recombine the light in the two interferometer arms, with one arm including a retroreflector on a delay stage with $100 \mathrm{~nm}$ step resolution. A tuneable bandpass filter is applied to select the emission from either the coupled or the uncoupled QDs. The interferogram was recorded as real-space images of the recombined and focused detection beams on a camera.

Optical properties of superfluorescence, superradiance, and subradiance. As shown in Figure $3 b$, we observed that the PL decay of the SF state is initially very fast and cannot be described with a single exponential because the decay rate is dependent on the number of excited TLS, $\Gamma(N) \sim N$, and therefore decreases during the decay. Consequently, the SF decay rate should converge towards the decay rate of the uncoupled nanocrystals. However, we observe that the SF decay trace crosses the bi-exponential PL decay of the uncoupled QDs after $97 \%$ of the photons are emitted due to long decay components. These long decay components might originate from coupled QDs where the individual dipoles are out of phase and interfere destructively, known as subradiance $(S B R)^{29,34}$. In ensembles with inhomogeneously broadened PL, SF and subradiant states can coexist, and we find a good agreement of the predicted excited state population with the measured PL decay ${ }^{35}$.

An out-of-phase coupling amongst the QDs is expected to result in a higher photon energy of the subradiant state compared to the SF state. In Extended Data Figure 7 and Extended Data Figure 9, we provide an analysis of the dynamical energy shift observed at high excitation power density for $\mathrm{CsPbBr}_{3}$ and $\mathrm{CsPbBr}_{2} \mathrm{Cl}$ QD superlattices, respectively. Examples of emission spectra at different times are reported in Extended Data Figure 7a and Extended Data Figure 9a for the respective QD halide compositions. In Extended Data Figure $7 \mathrm{~b}$ and Extended Data Figure 9b, we plot the fitted centre photon energy of time-sliced PL spectra (2 ps bin) as a function of the fitted peak area (i.e., the time-dependent emission intensity), as obtained from excitation power-dependent streak camera images, again for both, $\mathrm{CsPbBr}_{3}$ and $\mathrm{CsPbBr}_{2} \mathrm{Cl}$ QD superlattices. This effectively shows the energetic shift of the SF state as a function of its occupation, with the different curves representing different initial excitation powers. The green arrows indicate the time sequence of the individual analysed spectral 
traces. By increasing the excitation power, we observe that the initial dynamical red-shift is

414 the largest for the highest excitation power, as is expected from its relationship to the number

415 of excited coupled QDs. Hence, when the number of excited coupled QDs decreases during 416 the decay process, the emission energy blue-shifts to higher energy, as can be seen in 417 Extended Data Figures 7c and 9c where the fitted centre photon energy is plotted as a 418 function of time. We observe the most pronounced energetic blue-shift for the highest excitation power, resulting in a final emission with a photon energy that has been boosted incrementally more in comparison to the blue-shift for low excitation power, which is another indication of the presence of subradiant states that emit at higher energies. For high excitation power, the SF state becomes depopulated much faster since more QDs are coupled simultaneously. Then, at long timescales after the initial decay, the percentage of subradiant states becomes dominant, resulting in a blue-shift of the PL emission.

Superfluorescence fit model. SF decay traces as in Figure $3 b$ cannot be fitted well with mono- or bi-exponential functions because the decay rate is proportional to the number of excited coupled QDs $\Gamma(N) \sim N$, which also decays over time. Furthermore, the resulting characteristic decay neither exactly follows stretched-exponential nor a power-law dependence $^{36}$, whereas the PL decay of the uncoupled QDs is well described by a biexponential behaviour, where the initial fast decay $\tau_{\mathrm{QD}}=349.8 \pm 0.4$ ps accounts for over $96 \%$ the total emitted photons. Nevertheless, we found that the best approximate fit to the SF decay trace is the Kohlrausch stretched-exponential decay model $\frac{f(t)}{f(0)}=\exp \left[-\left(\Gamma_{\text {stretched }} \cdot t\right)^{\beta}\right]$, where $\Gamma_{\text {stretched }}$ is the average decay time and $\beta \in[0,1]$ is the stretch parameter, which represents the distribution of decay rates ${ }^{37}$. Using this model to fit the SF decay curve, we obtain an average decay time $\tau_{\text {stretched }}=40.4 \pm 0.5$ ps and a stretch parameter $\beta=0.457 \pm$ 436 0.002 .

437 At a high excitation density, as shown in Figure 5b for $\mathrm{CsPbBr}_{3} \mathrm{QD}$ superlattices and in Extended Data Figure 8b for $\mathrm{CsPbBr}_{2} \mathrm{Cl}$ QD superlattices, we observe oscillations in the decay. To model the SF decay with this characteristic ringing behaviour, we used a decay model consisting of a bi-exponential decay that is multiplied by a damped oscillating term 441 $1+B \cdot \exp \left(-\gamma_{\mathrm{Damp}} t\right) \cdot \cos \left(\omega t+\phi_{0}\right)$. Furthermore, for the rising edge of the emitted pulse we 
442 take into account a Gaussian rise term $\sim \exp \left[-\left(\frac{t-\tau_{\mathrm{D}}}{\tau_{\text {rise }}}\right)^{2}\right]$, such that the complete fit function is 443 given by (ref. $^{38}$ ):

$$
\begin{aligned}
f(t)=\sum_{n=1,2} A_{n} \cdot \exp \left(\frac{\tau_{\text {rise }}^{2}}{4 \tau_{n}^{2}}-\frac{t-\tau_{D}}{\tau_{n}}\right) \\
\cdot\left[\frac{1}{2}\left(1+B \cdot \exp \left(-\gamma_{\text {Damp }}\left(t-\tau_{\mathrm{D}}\right)\right) \cdot \cos \left(\omega\left(t-\tau_{\mathrm{D}}\right)+\phi_{0}\right)\right)\right. \\
\left.\cdot\left[1+\operatorname{erf}\left(\frac{t-\tau_{D}}{\tau_{\text {rise }}}-\frac{\tau_{\text {rise }}}{2 \tau_{n}}\right)\right]\right]
\end{aligned}
$$

444

Here, $A_{n}$ are the amplitudes of the exponential decay with the corresponding decay time constants, $\tau_{n}$. Both the fast decay time and the long decay time component (Extended Data Figure 7d for $\mathrm{CsPbBr}_{3}$ QD superlattices and Extended Data Figure 9d for $\mathrm{CsPbBr}_{2} \mathrm{Cl}$ QD superlattices) decrease upon increasing the excitation density, whereas the rise time, $\tau_{\text {rise }}=2.4 \pm 0.3 \mathrm{ps}$ for $\mathrm{CsPbBr}_{3}$ QD superlattices $\left(\tau_{\text {rise }}=3.4 \pm 1.0 \mathrm{ps}\right.$ for $\mathrm{CsPbBr}_{2} \mathrm{Cl}$ QD superlattices), stays approximately constant (probably clamped by the time resolution of the setup). In the upper panel of Figure $5 \mathrm{c}$, we plot the power-dependent effective decay time $\tau_{\mathrm{SF}}=\frac{A_{1} \tau_{1}+A_{2} \tau_{2}}{A_{1}+A_{2}}$ for $\mathrm{CsPbBr}_{3} \mathrm{QD}$ superlattices, where $\tau_{1}, \tau_{2}$ are the decay times of the biexponential fit and $A_{1}, A_{2}$ the corresponding amplitudes, which was fitted with $\tau_{\mathrm{SF}}(P)=\frac{\tau_{\mathrm{QD}}}{\zeta \cdot P+1}+$ $y_{0}$, with a fixed value of $\tau_{Q D}=400 \mathrm{ps}$, obtained from the time-resolved PL measurements of uncoupled QDs and an additional offset $y_{0}$ to account for effects like the finite time resolution. We obtain good agreement with the expected behaviour $\left(\tau_{\mathrm{SF}} \sim \tau_{\mathrm{QD}} / N\right)$ for a value $\zeta_{\mathrm{CSPbBr} 3}=$ $0.29 \pm 0.04 \frac{\mathrm{cm}^{2}}{\mu \mathrm{J}}$. In the lower panel of Figure $5 \mathrm{c}$, we plot the delay time $\tau_{D}$ for the $\mathrm{CsPbBr}_{3} \mathrm{QD}$ superlattices as a function of the excitation power. In our analysis, the delay time is composed of the actual delay time due to the SF build-up and a systematic, constant time-offset because the absolute arrival time of the excitation pulse (which has a different wavelength than the emission) at the sample cannot be measured reliably at the required precision from the streak camera data. We observe a decrease in $\tau_{\mathrm{D}}$ of $\sim 6 \mathrm{ps}$ when increasing the excitation density by almost 2 orders of magnitude. We have fitted this behaviour with $\tau_{\mathrm{D}}=y_{\text {offset }}+A \cdot \ln \left(\zeta P_{\mathrm{Exc}}+\right.$ 1) $/\left(\zeta P_{\mathrm{Exc}}+1\right)$ because we assume that $\tau_{\mathrm{D}} \sim \ln (N) / N$ and that the number of excited coupled 
emitters $N \sim \alpha P_{\text {Exc }}+1$ is proportional to the excitation power. Herein, we use a fixed value $\zeta_{C S P B B r 3}=0.29 \pm 0.04 \frac{\mathrm{cm}^{2}}{\mu \mathrm{J}}$, which we obtained from the fit of the effective decay in the upper panel of Figure $5 \mathrm{c}$. The resulting fit agrees very well with the data. To obtain the absolute time delay, we subtracted the constant offset $y_{\text {offset }}$ of the time-delay fit from the time-delay data points. SF occurs when $\sqrt{\tau_{\mathrm{SF}} \tau_{\mathrm{D}}}<T_{2}^{*}$, where $T_{2}^{*}$ is the exciton pure dephasing time, whereas $\sqrt{\tau_{\mathrm{ASE}} \tau_{\mathrm{D}}}>T_{2}^{*}$ signifies the amplified spontaneous emission (ASE) regime, when $\tau_{\mathrm{ASE}}$ is the decay time (ref. ${ }^{33}$ ). Considering that the coherence time $T_{2}<T_{2}^{*}$ extracted from the full-width at half-maximum of single QDs ${ }^{11}$ is of the order of $T_{2}=6.6 \mathrm{ps}$, our measurements reveal a fast decay of $\sim 14$ ps and a delay time of $<1$ ps which satisfies the criterion for the appearance of SF.

A similar analysis was performed on $\mathrm{CsPbBr}_{2} \mathrm{Cl} \mathrm{QD}$ superlattices as shown in Extended Data Figure 8. In the upper panel of Extended Data Figure 8c we plot powerdependent effective decay time and fit the data with the same model as described above, using $\tau_{Q D}=250$ ps and we obtain a value $\zeta_{C S P B B r 2 C l}=0.08 \pm 0.01 \frac{\mathrm{cm}^{2}}{\mu \mathrm{J}}$. The peak intensity of the decay curves as a function of the excitation density is shown in the middle panel of the Extended Data Figure 8c, which increases super-linearly with a power-law dependence with an exponent $\alpha_{C S P B B r 2 C l}=1.3 \pm 0.1$. Also the delay time decreases as a function of the excitation density as displayed in the lower panel of Extended Data Figure $8 \mathrm{c}$ and fit the data. Again, we fit the data using the same formula for $\tau_{D}$ as described above with a fixed value $\zeta_{C S P B B r 2 C l}=0.08 \pm 0.01 \frac{\mathrm{cm}^{2}}{\mu \mathrm{J}}$ and obtain good agreement.

\section{Additional references}

34 DeVoe, R. G. \& Brewer, R. G. Observation of Superradiant and Subradiant Spontaneous Emission of Two Trapped lons. Physical Review Letters 76, 2049-2052 (1996).

35 Bienaimé, T., Piovella, N. \& Kaiser, R. Controlled Dicke Subradiance from a Large Cloud of Two-Level Systems. Physical Review Letters 108, 123602 (2012).

36 Guerin, W., Rouabah, M. T. \& Kaiser, R. Light interacting with atomic ensembles: collective, cooperative and mesoscopic effects. Journal of Modern Optics 64, 895-907 (2017).

37 van Driel, A. F. et al. Statistical analysis of time-resolved emission from ensembles of semiconductor quantum dots: Interpretation of exponential decay models. Physical Review B 75, 035329 (2007).

38 Naeem, A. et al. Giant exciton oscillator strength and radiatively limited dephasing in two-dimensional platelets. Physical Review B 91, 121302 (2015). 


\section{Acknowledgements}

501 We thank D. J. Norris, C. Schwemmer, D. Urbonas, and F. Scafirimuto for helpful 502 discussions. F. Krieg is acknowledged for providing additional samples for control 503 experiments. M.A.B., M.V.K., T.S., R.F.M., and G.R. acknowledge the European Union's 504 Horizon-2020 programme through the Marie-Sklodowska Curie ITN network PHONSI (H2020MSCA-ITN-642656) and the Swiss State Secretariat for Education Research and Innovation (SERI). M.I.B. acknowledges financial support from the Swiss National Science Foundation (SNF Ambizione grant, Grant No. PZENP2_154287). M.V.K. acknowledges financial support from the European Research Council under the European Union's Seventh Framework Program (FP/2007-2013) / ERC Grant Agreement No. 306733 (NANOSOLID Starting Grant).

\section{Author contributions}

512 The work originated from ongoing interactions between G.R., M.V.K, R.F.M., and T.S. M.I.B. 513 prepared the samples and performed their structural characterization. G.R., M.A.B., and T.S. 514 performed the optical experiments, interpreted the data with input from R.F.M. G.R. and 515 M.A.B. wrote the manuscript with input from all the co-authors. R.F.M., M.V.K., and T.S. 516 supervised the work.

517

\section{Author information}

519 Correspondence and requests for materials should be addressed to G.R. (rainog@ethz.ch), 520 M.V.K. (mvkovalenko@ethz.ch), and T.S. (tof@zurich.ibm.com).

Data availability. The data that support the findings of this study are available from the 523 corresponding authors upon reasonable request.

The authors declare no competing interests. 
Extended Data Figure 1 | Quantitative analysis of $\mathrm{CsPbBr}_{3} \mathrm{NC}$ size distribution. a, Low-resolution TEM image of the NC material which is used to prepare the superlattices. $\mathbf{b}$, Histogram of NC sizes (of $>100$ NCs) as obtained from TEM image analysis. The solid line is a fit with a normal distribution.

Extended Data Figure 2 | HAADF-STEM image of a single superlattice of $\mathrm{CsPbBr}_{3} \mathbf{N C s}$. Individual NCs (bright spots in the image) are well-resolved.

Extended Data Figure 3 | PL excitation measurement of $\mathrm{CsPbBr}_{3}$ QD superlattices. Using a weak, tuneable excitation source, we plot the PL intensity (black circles) obtained at $2.30 \mathrm{eV}$ photon energy as a function of excitation photon energy. The shaded areas are Gaussian peak fits. This shows that the coupled QD feature is also present in the absorption spectrum, in addition to the uncoupled QDs and more energetically higher states.

Extended Data Figure 4 | Power dependent PL properties of $\mathrm{CsPbBr}_{3}$ QD superlattices. a, Colour-coded $\mathrm{PL}$ emission in the low-power excitation regime, shown for increasing excitation fluence of $10 \mathrm{~nJ} / \mathrm{cm}^{2}$ (light green), $60 \mathrm{~nJ} / \mathrm{cm}^{2}$ (light blue), $150 \mathrm{~nJ} / \mathrm{cm}^{2}$ (yellow), $310 \mathrm{~nJ} / \mathrm{cm}^{2}$ (dark green) and $600 \mathrm{~nJ} / \mathrm{cm}^{2}$ (dark blue). b, PL intensity integrated over the spectral emission range of the uncoupled QDs (blue circles) and coupled QDs (red circles) in a log-log plot and the total emitted intensity (yellow circles). Fits to the data reveal a perfectly linear behaviour, as represented by a fitted power-law exponent $m$ of 1 . c. Colour-coded PL emission in the highpower excitation regime, shown for increasing excitation fluence of $330 \mu \mathrm{J} / \mathrm{cm}^{2}$ (light green), $1270 \mu \mathrm{J} / \mathrm{cm}^{2}$ (light blue), $2130 \mu \mathrm{J} / \mathrm{cm}^{2}$ (yellow), $3470 \mu \mathrm{J} / \mathrm{cm}^{2}$ (dark green) and $6330 \mu \mathrm{J} / \mathrm{cm}^{2}$ (dark blue). d, PL intensity integrated over the spectral emission range of the uncoupled QDs (blue) and coupled QDs (red) in a log-log plot and the total emitted intensity (yellow). Fits to the data reveal a power-law behaviour with a linear increase for the SF emission, a slightly sublinear increase for the uncoupled QDs and a less sublinear increase for the total emitted intensity.

Extended Data Figure 5 | Gaussian first-order coherence decay and photon bunching in pulsed excitation of $\mathrm{CsPbBr}_{3}$ QD superlattices. a, First-order coherence of the coupled QD emission extracted from the fringe contrast of the interferograms as a function of differential delay time between the arms of a Michelson interferometer, revealing a mixture of Gaussian (Kubo) and exponential decay (for some of the superlattices). b, Second-order photon correlation measurement of the coupled QD emission from a single superlattice showing photon bunching at zero delay under pulsed excitation with a $40 \mathrm{MHz}$ repetition rate.

Extended Data Figure 6 | SF in $\mathrm{CsPbBr}_{2} \mathrm{Cl}$ QD superlattices. a, Band-pass filtered PL spectra of uncoupled QDs (blue) and coupled QD emission (red) of $\mathrm{CsPbBr}_{2} \mathrm{Cl}$ perovskite superlattices. $\mathbf{b}$, Second-order photon correlation measurement of uncoupled QDs (top panel, $g^{(2)}(\tau)=1$ ) showing flat correlation function, and the coupled QD emission peak band (lower panel, $g^{(2)}(0)=1.15$ ) showing photon bunching.

Extended Data Figure 7 | SF decay and dynamic red-shift of $\mathrm{CsPbBr}_{3}$ QD superlattices. a, PL spectra (integrated over 2 ps time window) at different time delays in a semi-log scale. $\mathbf{b}$, The PL spectra are fitted to a single Gaussian peak function, and the fitted peak amplitude as a function of the emission energy is plotted for various excitation densities. Green arrows indicate the time evolution of the emission peak. The black dashed line denotes the mean energy at the lowest excitation density, and the grey shaded area is the peak's FWHM. c, Fitted peak centre energy as a function of time for various excitation densities. d, Fast and slow PL decay time 
components $\tau_{1}$ and $\tau_{2}$ of the SF bi-exponential fit model as a function of excitation density in a semi-log plot. The error bars represent the parameters' fit uncertainty.

Extended Data Figure 8 | Burnham-Chiao ringing behaviour in $\mathrm{CsPbBr}_{2} \mathrm{Cl}$ QD superlattices. a, Streak camera image of SF dynamics obtained with high excitation density of $1600 \mu \mathrm{J} / \mathrm{cm}^{2}$. b. Extracted time-resolved emission intensity traces for three different excitation powers. Solid lines are best-fits to a model that employs a bi-exponential decay function with damped oscillations. c, Top: Effective SF decay (blue circles) as a function of the excitation power density fitted according to the SF model (solid blue line). Middle: Red circles represent the peak SF emission intensity that increases super-linearly with excitation power, corresponding to a power-law dependence with an exponent $\alpha=1.3 \pm 0.1$ (solid dark-red line). Bottom: The extracted delay time $\tau_{\mathrm{D}}$ (green circles) decreases at high excitation power due to the increased interaction among the emitters. The green solid line is the best fit according the model described in the Methods section. The error bars represent the parameters' fit uncertainty.

Extended Data Figure 9 | SF decay and dynamic red-shift of $\mathrm{CsPbBr}_{2} \mathrm{Cl}$ QD superlattices. a, PL spectra (integrated over 2 ps time window) at different time delays in a semi-log scale. b. The PL spectra are fitted to a single Gaussian peak function, and the fitted peak amplitude as a function of the emission energy is plotted for various excitation densities. Green arrows indicate the time evolution of the emission peak. The black dashed line denotes the mean energy at the lowest excitation density, and the grey shaded area is the peak's FWHM. c, Fitted peak centre energy as a function of time for various excitation densities. d, Fast and slow PL decay time components $\tau_{1}$ and $\tau_{2}$ of the SF bi-exponential fit model as a function of excitation density. The error bars represent the parameters' fit uncertainty.

Extended Data Figure 10 | PL decay from a control sample of diluted $\mathrm{CsPbBr}_{3}$ QD. a, Streak camera measurement of a control sample prepared by spin-coating a low concentration of $\mathrm{CsPbBr}_{3} \mathrm{QD}$ dispersed in polystyrene such that no QD coupling is realized. The excitation conditions were comparable to Figure 5 , using an excitation density of $1600 \mu \mathrm{J} \mathrm{cm}^{-2}$. The comparably long decay requires that on the streak camera a longer time range with lower temporal resolution compared to the measurement in Figure 5 has to be used. The absence of dynamic red-shift, accelerated decay and ringing proves that the observed SF features cannot be explained by single QD physics but are a multi-particle effect. b, Time-resolved PL trace (blue) obtained from this streak data. For direct comparison, the equivalent data from a superlattice sample showing SF (Figure 5b, $1200 \mathrm{\mu} \mathrm{cm}^{-2}$ ) is shown (red). Inset: Instrument response function of the streak camera. Using the same instrument settings as for the superlattices measurement, the temporal response of the streak camera using scattered light from a $100 \mathrm{fs}$ laser pulse at $400 \mathrm{~nm}$ gives a Gaussian-shaped instrument response with a FWHM of $4.3 \mathrm{ps}$ (Gaussian fit is shown as red solid line). 


\section{Establish coherence via photon field}

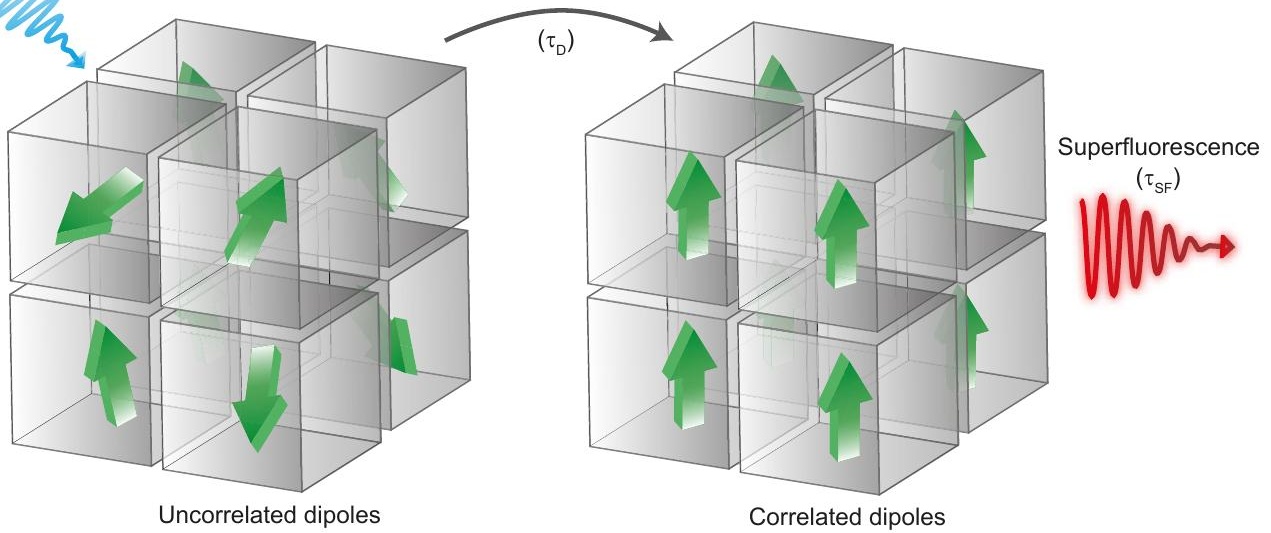




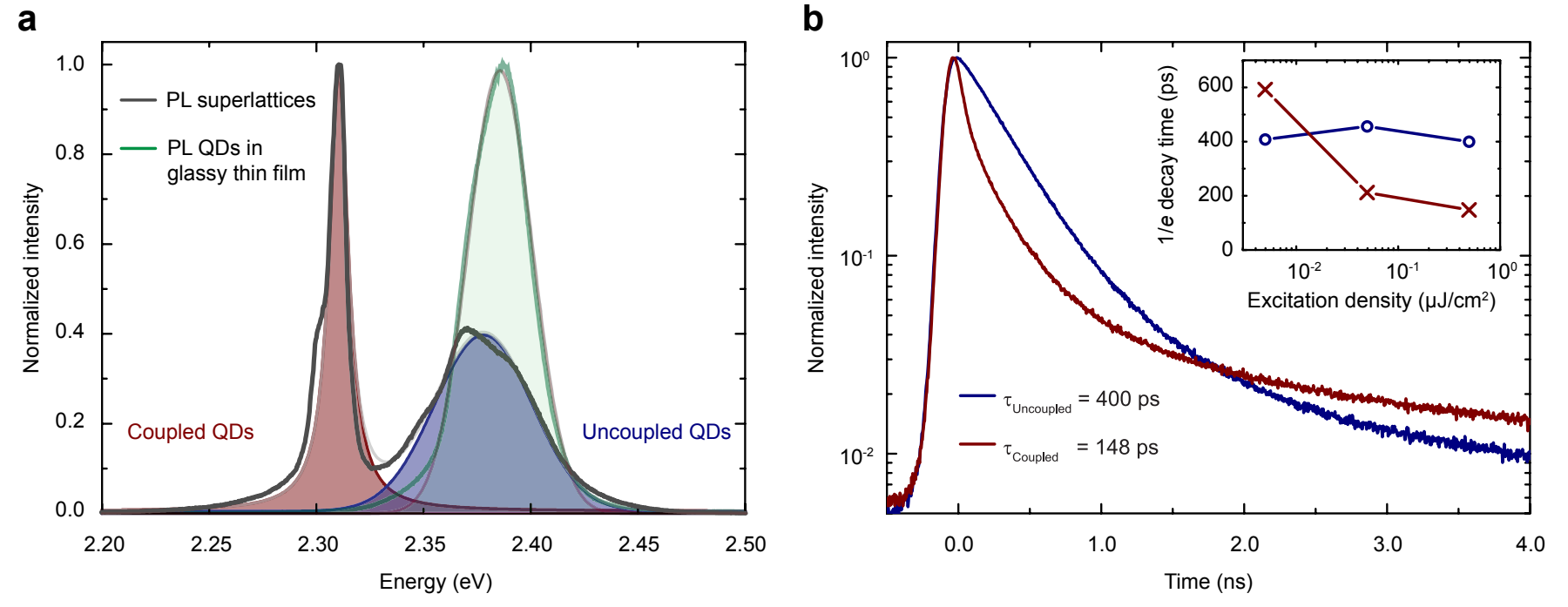



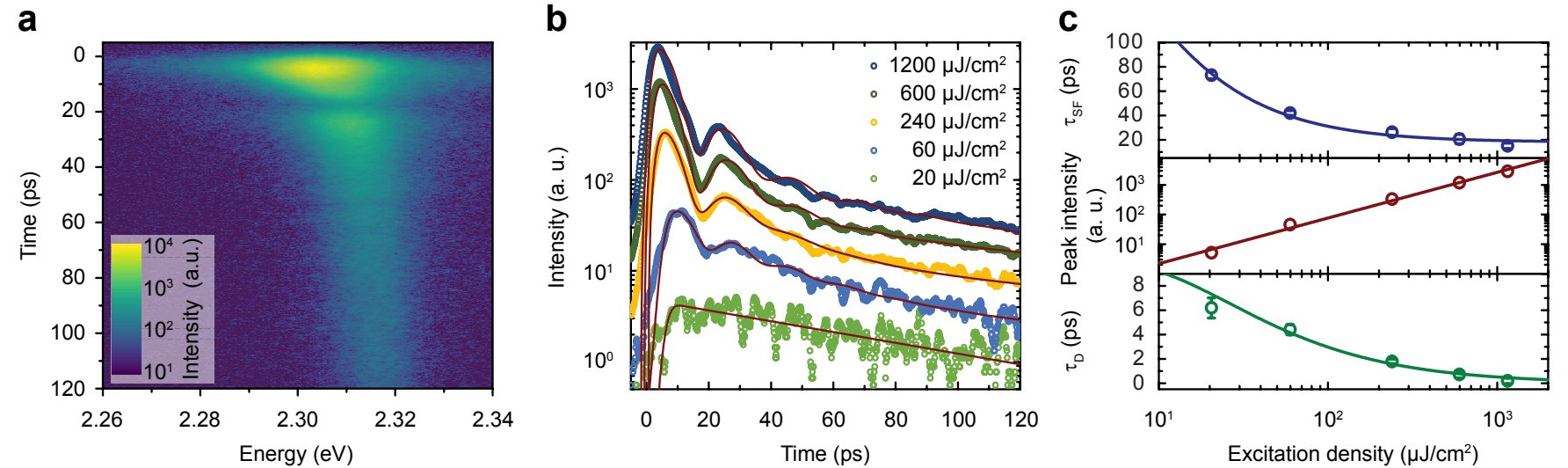